\title{
Creep of concrete under uniaxial and biaxial stresses at elevated temperatures*
}

\author{
by S. Arthanari, BE, MSc(Eng), PhD and C. W. Yu, PhD, DIC, MASCE, AMICE
}

\author{
Contribution by J. Timusk \\ University of Toronto: Department of Civil Engineering
}

\begin{abstract}
Of the more than a thousand references in the literature to creep of concrete, only a mere handful deal with measurements of creep in the unloaded direction, and fewer still with creep under multiaxial loading. This one-sided approach to a volumetric phenomenon has been dictated primarily by the degree of accuracy required to obtain meaningful results pertaining to Poisson's creep effects. Here the accuracy needs to be at least five times higher than in the case of uniaxial loading.
\end{abstract}

It is unfortunate that the authors did not incorporate into their rigs essential features used in the work carried out by Ross (their reference 3 ), viz. springs and a means of measuring the actual load applied to the concrete. Our calculations, based on dimensions scaled from Figure 2, indicate that about $10 \%$ of the prestress is lost when the load is transferred from the jack to the nuts via the flexible distributing plate. A further $8 \%$ can be lost to creep if the frame is restressed 1 day after the first loading. Also an estimated 30 to $40 \%$ change in the applied stress, due to temperature effects, could occur because the tensioning rods are in a box where temperature is not controlled. When the asbestos boards were removed for strain measurement, the rod temperature would tend to approach room temperature. With the panels closed, the interior of the asbestos box is heated by the uninsulated sides of the loading platens and the distance pieces. With a temperature differential of 60 $\operatorname{degC}$ in the $80^{\circ} \mathrm{C}$ tests, a temperature fluctuation of 30 to $40 \mathrm{degC}$ in the box enclosing the loading rig could easily take place.

Heating of the specimens for $24 \mathrm{~h}$ before loading would have pre-dried the concrete in the unsealed specimens, thereby eliminating much of the creep and shrinkage. This procedure is quite the reverse of what would be encountered in practice where a nuclear pressure vessel is prestressed before being subjected to high temperatures. Would this pre-drying of the unsealed specimens not explain the controversial statement regarding ultimate creep of sealed specimens?

* Pages 149 to 156 of Magazine No. 60.
Could not the "immediately after loading" specific creep strains in Figures 5, 6,7 and 8 be explained by a lowering of the elastic modulus of the dried and heated concrete in relation to the room temperature tests used for the determination of the elastic constants?

It would also be of considerable interest to know how Poisson's ratios from uniaxial sustained load tests compared with those shown in Figure 10.

\section{Reply by the authors}

The authors wish to thank Professor Timusk for his comments. Because of the length of the article, the loading technique had not been described in detail. In fact, in applying the load to the specimens, calibrated pressure gauge and torque spanners were used to ensure a minimum loss of prestress due to transfer. Further, the Macalloy bars were rested on the ground and thus exposed to ambient conditions all the time. Therefore the bars were always in an equilibrium condition with regard to temperature. The time needed for a reading to be taken could not be more than 2 to 3 minutes, and the effect on the temperature of the bars could only be negligible.

The $24 \mathrm{~h}$ pre-heating was necessary because of the difficulty of attaining the correct temperature in the specimens. Since the long-term effect was the main parameter under study, the adopted experimental technique was considered acceptable. Also, the conclusion that sealed specimens exhibited greater ultímate creep was made from the trend of the rate of creep and not from the magnitude of creep observed. The $24 \mathrm{~h}$ pre-heating would certainly not effect this conclusion.

It should be pointed out that creep strain occurs on loading even at ambient temperatures and the "immediate strains' are greater than the elastic strains. This phenomenon is amplified at high temperatures and prolonged application of loading. The reduction of elastic modulus on heating is considered too small to be regarded as an explanation. 